\title{
INDICADORES DE RIESGO PSICOSOCIAL EN FUNCIÓN DE LOS PUESTOS DE TRABAJO
}

\section{PSYCHOSOCIAL RISK INDICATORS ACCORDING TO THE PLACE OF JOBS}

\author{
Cuesta-Mero Giovani ${ }^{1 *}$; Andrade-Bastidas Paulo²; Sánchez-Arteaga Alex ${ }^{3}$ \\ ${ }^{1}$ Universidad Internacional de la Rioja, UNIR. La Rioja, España. \\ 'Universidad Internacional de la Rioja, UNIR. La Rioja, España. \\ ${ }^{3}$ Universidad de Especialidades Espíritu Santo, UEES. Guayaquil, Ecuador.
}

*Correo: giodar-cu-me49@gmail.com

\begin{abstract}
Resumen
El presente trabajo es un manuscrito de revisión bibliográfica de los indicadores de riesgo psicosocial que se encuentran presentes en los trabajadores. La importancia de evaluar los riesgos psicosociales, radica en que su naturaleza está intrínsicamente relacionada con afectaciones prolongadas en los trabajadores si estos no son controlados adecuadamente. Los trabajadores que se sienten expuestos a riesgos psicosociales, tales como sobrecarga de trabajo, tareas repetitivas o tareas de muy corta duración, exceso de carga mental, entre otros, son aquellos que tienen porcentajes de respuestas de sintomatología psicosomática más elevados. Una vez revisadas las estadísticas de los factores de riesgo psicosocial, podemos notar que los mismos, afectan a un porcentaje significativo de la población trabajadora que fue muestreada en un estudio realizado por el Instituto Nacional de Seguridad e Higiene en el Trabajo (España), por lo cual, es necesario evaluar en cada centro de trabajo si estos factores están presentes en la organización, para de esta forma, garantizar al trabajador un adecuado bienestar en el trabajo, derecho adquirido según normativas legales tanto nacionales, como internacionales. Finalmente, debe considerarse que no todos los factores de riesgo psicosocial, están presentes en todos los puestos de trabajo, sino que existen puestos de trabajo en donde un factor puede estar más arraigado que otro.
\end{abstract}

Palabras clave: trabajador, seguridad, salud, riesgo laboral, riesgo psicosocial.

\begin{abstract}
The present work is a bibliographic review manuscript of the psychosocial risk indicators that are present in the workers. The importance of evaluating psychosocial risks lies in the fact that their nature is intrinsically related to prolonged effects on workers if they are not adequately controlled. Workers who feel exposed to psychosocial risks, such as work overload, repetitive tasks or tasks of very short duration, excessive mental load, among others, are those who have higher percentages of responses to psychosomatic symptoms. Once the statistics on psychosocial risk factors were reviewed, we can see that they affect a significant percentage of the working population that was sampled in a study carried out by the National Institute for Safety and Hygiene at Work (España). Therefore, it is necessary to evaluate in each workplace if these factors are present in the organization, in order to guarantee the worker an adequate well-being at work, a right acquired under both national and international legal regulations. Finally, it must be considered that not all psychosocial risk factors are present in all jobs, but there are jobs where one factor may be more ingrained than another.
\end{abstract}

Keywords: worker, safety, health, occupational risk, psychosocial risk.

Información del manuscrito:

Fecha de recepción: 29 de abril de 2019

Fecha de aceptación: 20 de junio de 2019

Fecha de publicación: 10 de julio de 2019 


\section{Introducción}

Desde el punto de vista legal, el artículo 16.2 de la Ley de Prevención de Riesgos Laborales (Real Decreto Ley 31/1995), manifiesta que "los instrumentos esenciales para la gestión y aplicación del plan de prevención de riesgos, (...) son la evaluación de riesgos laborales y la planificación de la actividad preventiva", por lo que es de obligado cumplimiento que en los centros de trabajo, se realicen evaluaciones periódicas de los riesgos para la seguridad y salud de los trabajadores, además de que si las mismas manifestaran que los trabajadores están expuestos a riesgos no tolerables, se deben realizar actividades preventivas para poder eliminarlos o reducirlos.

Al referirse a evaluación de riesgos, están contemplados todos aquellos que pudieran suscitarse según la naturaleza de cada actividad, por ejemplo, riesgos mecánicos, físicos, biológicos y además la evaluación del riesgo psicosocial (Bernal et al., 2015).

Los riesgos psicosociales, según Guilguiruca et al., "son hechos, situaciones o contextos con una clara probabilidad de dañar la salud física, social o mental del trabajador de forma importante" (2015, p. 59). Estas condiciones, "presentes en una situación laboral, están relacionadas con la organización del trabajo, contenido del puesto, la realización de la tarea e incluso su entorno" (Gil-Monte, 2009, p. 169).

Por lo tanto, la importancia de evaluar los riesgos psicosociales, radica en que su naturaleza está intrínsicamente relacionada con afectaciones prolongadas en los trabajadores si estos no son controlados adecuadamente (Palomo-Vélez et al., 2015); por lo cual el presente trabajo, se estructura basándose en la evaluación de los factores de riesgo psicosocial.

Por consiguiente, el presente trabajo tiene como objetivo revisar y discutir la literatura científica relacionada con los indicadores relativos a los factores de riesgo psicosocial.

\section{Riesgos psicosociales}

Los riesgos psicosociales, pueden deteriorar la salud de los trabajadores mientras desempeñan sus labores, o inclusive fuera de su trabajo (Gil-Monte, 2009). Estos riesgos psicosociales, junto con sus 
factores de riesgo, se pueden definir como factores organizacionales que además de afectar negativamente a su salud, pueden provocar estrés (Martín-García et al., 2015), siendo este una de las principales causas de enfermedades y accidentes en el trabajo (Gil-Monte, 2009).

Los trabajadores que se sienten expuestos a riesgos psicosociales, tales como sobrecarga de trabajo, tareas repetitivas o tareas de muy corta duración, exceso de carga mental, entre otros, son aquellos que tienen porcentajes de respuestas de sintomatología psicosomática más elevados, como por ejemplo problemas de sueño, cansancio, dolores de cabeza, mareos, entre otros (Gil-Monte, 2009).

Esta sintomatología psicosomática producto del estrés laboral, es reconocida como una epidemia dentro del trabajo, que ha sido derivada de las transformaciones que esta ha sufrido (Guilguiruca et al., 2015), como por ejemplo los cambios técnicos u organizativos en las empresas, cambios socioeconómicos, políticos 0 demográficos, sin dejar atrás a la globalización (Gil-Monte, 2009). Todas estas transformaciones se han suscitado a partir de la década de los 80, cuyas características tales como una fuerte intensificación, precarización e inestabilidad laboral fueron producto de varias estrategias encaminadas a impulsar una mayor adaptación de las organizaciones a partir de la productividad (Guilguiruca et al., 2015).

Según Guilguiruca et al. (2015), este escenario ha suscitado cambios en la esfera socio simbólica de la relación persona-trabajo, dando como resultado consecuencias tanto a nivel físico como mental que además de la sintomatología mencionada anteriormente, produce cambios en el entorno laboral, tales como ausentismos, baja satisfacción, acoso laboral, resistencia a los cambios, entre otras.

Todo esto producto de que las situaciones de la vida laboral, se convierten en tan impredecibles, incontrolables o ambas a la vez, que el trabajador percibe inestabilidad tanto social, física y emocional.

Toda esta "carga mental" que los trabajadores tienen que llevar a cuestas, según Ceballos-Vásquez et al. (2015, p. 316), es: Un constructo multidimensional definido como la 
interacción entre las demandas cognitivas de la tarea (por ejemplo, memoria y atención), las características de la persona (por ejemplo, nivel educativo $y$ autoeficacia) y las características de la situación (por ejemplo, presión temporal). Entre sus causas cabe citar características de la tarea (por ejemplo, demandas de memoria y atención), presiones temporales y ritmo de trabajo, así como, las funciones a desempeñar, el grado de autonomía, y la interacción con otras personas.

Además de los factores laborales y personales de los que se ha hecho mención en lo que respecta a riesgo psicosocial, no hay que olvidar que la situación personal fuera del trabajo, también juega un papel muy importante en el desarrollo del incremento de los riesgos psicosociales. En este aspecto, García et al. (2016), hace referencia a que la situación personal es importante debido a que puede hacer sinergia en el impacto a la salud que un riesgo psicosocial pueda causar. Por ejemplo, en lo que respecta al género, el mismo autor señala que las mujeres quienes culturalmente tienen mayor responsabilidad del trabajo doméstico, tendrían una "doble jornada laboral" que se conoce como doble presencia, y también es un factor a ser analizado por las distintas metodologías de estimación de riesgo psicosocial.

\section{Indicadores de riesgo psicosocial}

Para establecer una idea clara y objetiva del impacto de los riesgos psicosociales en el trabajador, se tomarán en cuenta los indicadores relativos a los factores de riesgo psicosocial propuestos en la Encuesta Nacional de Condiciones de Trabajo $6^{\circ}$ EWCS - España (INSHT, 2017). Estos indicadores son:

3.1. Exigencias del Trabajo: rapidez, plazos o tareas simultáneas.

3.2. Ritmo de Trabajo.

3.3. Características de la Organización.

3.4. Trabajo directo con personas.

3.5. Grado de Autonomía.

3.6. Relaciones Sociales.

3.7. Percepción de la Inseguridad del empleo. 
3.8. Situaciones de violencia, acoso o discriminación.

\subsection{Exigencias del Trabajo}

En lo que respecta a las exigencias del trabajo, según la mencionada Encuesta Nacional (INSHT, 2017), del total de encuestados (3.364 trabajadores), el 34\% manifiesta que debe trabajar con plazos ajustados y el $33 \%$ manifiesta que debe trabajar a gran velocidad.

En la figura 1, se observan los porcentajes de trabajadores que manifiestan trabajar con plazos ajustados y a gran velocidad, clasificados según su situación profesional (asalariados y autónomos), edad (50 y más años, de 35 a 49 años y hasta 34 años) y ocupación (diversas ocupaciones), para de esta manera, establecer una idea del impacto que tienen las exigencias del trabajo, como factor de riesgo psicosocial a tener en cuenta en los trabajadores.

Por su parte, los trabajadores que presentan mayor índice de actividades laborales con plazos ajustados son los artesanos y trabajadores cualificados; mientras que, en las ocupaciones elementales, el $43 \%$ de los trabajadores trabaja a gran velocidad.

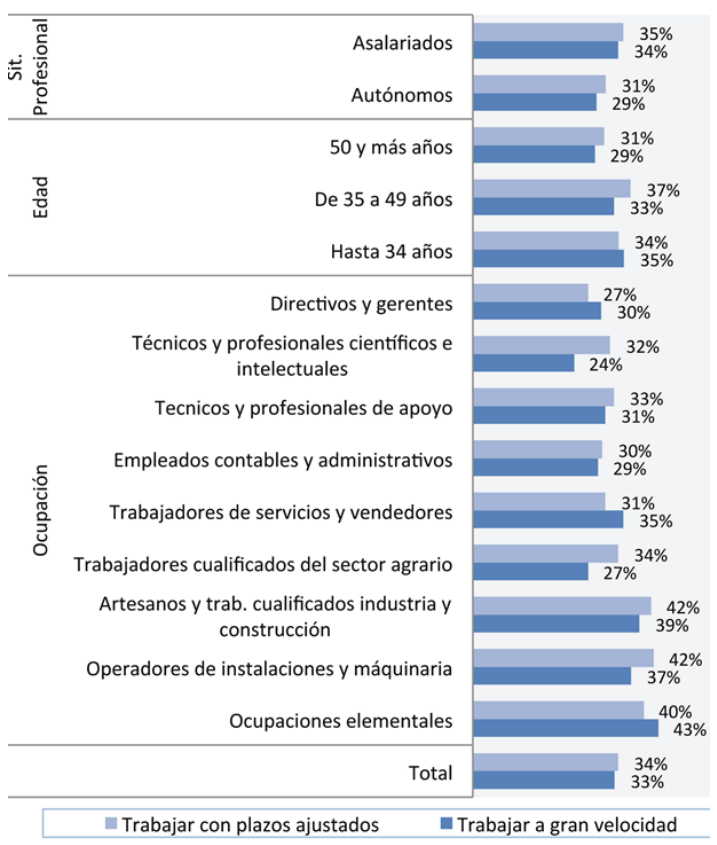

Figura 1. Intensidad del trabajo según la situación profesional, edad y ocupación (INSHT, 2017, p. 40)

\subsection{Ritmo del Trabajo}

El ritmo de trabajo es uno de los factores de riesgo que limita considerablemente la capacidad de un trabajador (INSHT, 2017), por lo cual según las estadísticas existen determinantes que condicionan el ritmo de trabajo. De esta manera, se concluye que las demandas directas de personas, tienen una mayor incidencia en el condicionamiento del ritmo de trabajo y su acumulación, ya que entre los años 2005 a 2015, se encuentra el 69\%, sobre una base de trabajadores de 3.364. Además, se denota que el 
ritmo de trabajo siempre se va a acelerar por uno o más factores y que esto afecta perennemente a más de un cuarto de los afectados.

\subsection{Características de la} Organización

En cuanto a las características de la organización, estas se refieren a aquellas políticas, metodologías (teletrabajo, uso de TIC's, entre otras), o maneras que tienen las organizaciones para trabajar (monotonía o trabajos repetitivos). Específicamente, se considera el cumplimiento del trabajador (siguiendo las distintas metodologías de trabajo) según las normas de calidad de la organización.

Según las estadísticas, se consideran las ocupaciones que se deben acomodar a normas de calidad precisas y la evaluación de la calidad del trabajo por el mismo trabajador. Por lo cual los artesanos y trabajadores de la industria $y$ cualificación son aquellos que más deben acoplarse a normas de calidad precisas (91\%), y los técnicos y profesionales de apoyo son aquellos quienes con más frecuencia (88\%), deben evaluar su propio trabajo. La muestra evaluada fue de 3.364 trabajadores.

\subsection{Trabajo directo con personas}

En lo que respecta al trabajo directo con personas, es muy común encontrar exigencias emocionales que permiten establecer un acercamiento con la persona en cuestión. Por ejemplo, si se trata de un trabajador que recibe reclamos, el mismo estará en contacto con personas que están disconformes con un producto o servicio, por lo cual se debe tener ciertas habilidades emocionales para tratar con personas que se encuentran en ese estado. Según las estadísticas expuestas en la figura 2 se demuestra que, sobre una base de 3.364 trabajadores, el $61 \%$ de los mismos, de hasta 34 años de edad, debe tratar con personas externas a la organización, así mismo el $49 \%$ de los trabajadores de entre 35 a 49 años y el $46 \%$ de los trabajadores de 50 y más años de edad.

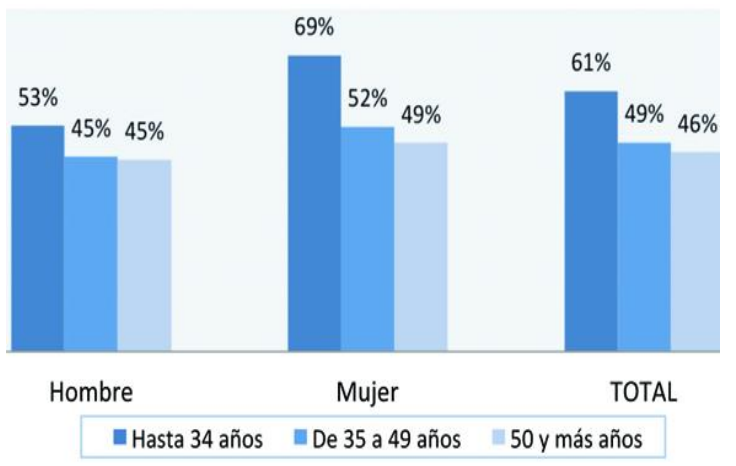

Figura 2. Relación directa con personas externas (INSHT, 2017, p. 43) 


\subsection{Grado de Autonomía}

Este indicador se refiere a la posibilidad de elegir las tareas, 0 cambiar el orden de las mismas. En este sentido sobre una base de 3.364 trabajadores, se evaluó la posibilidad de cambiar el orden de las tareas, el método de trabajo y la velocidad o ritmo de trabajo, por lo cual según las estadísticas de la figura 3, la mayoría de ocupaciones tiene un buen grado de autonomía, a excepción de los operadores de instalaciones o maquinarias que obtuvieron resultados de $35 \%$ a la autonomía de cambiar el orden de las tareas, $40 \%$ el método de trabajo y un $50 \%$ la velocidad o ritmo del mismo.

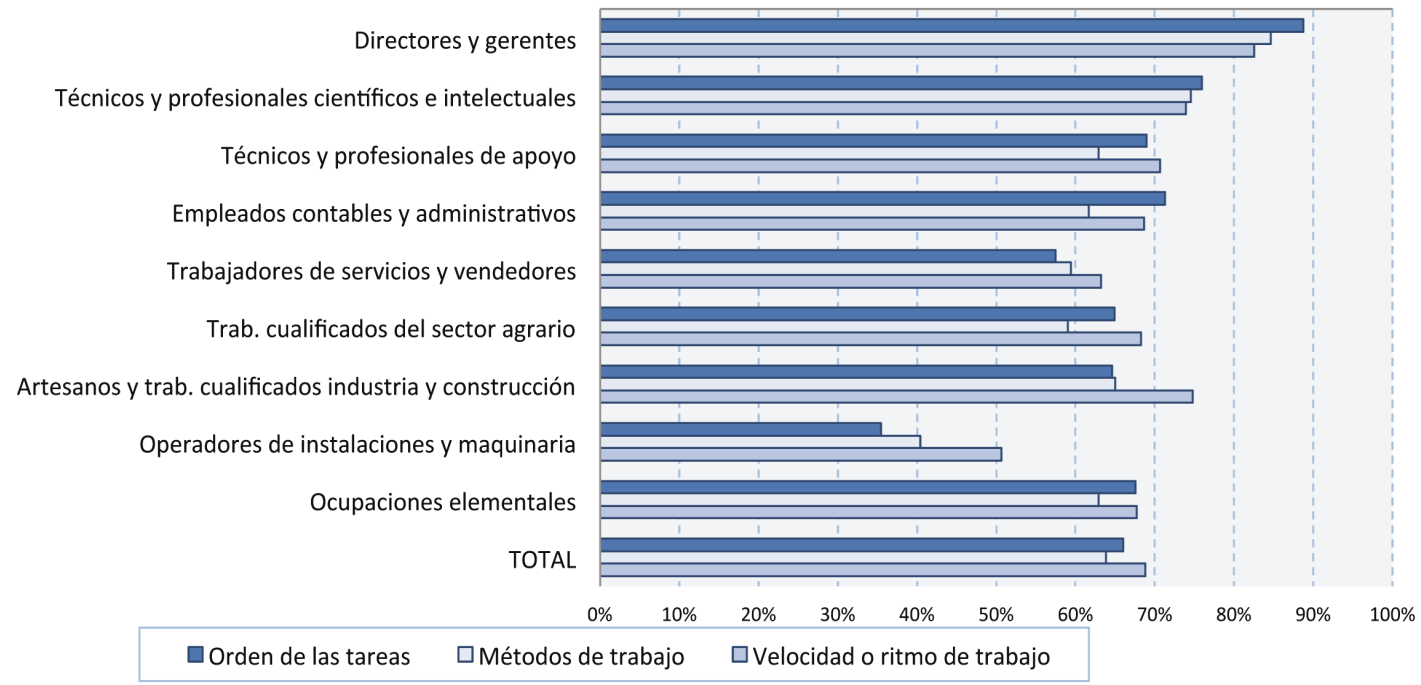

Figura 3. Autonomía por ocupación (INSHT, 2017, p. 45)

\subsection{Relaciones Sociales}

Las relaciones sociales desde el punto de vista del riesgo psicosocial, se miden en función de la falta de apoyo social en el trabajo por parte de jefes y compañeros. En este sentido, las estadísticas demuestran que, sobre una base de 2.776 trabajadores, el sector de trabajadores cualificados del sector agrario en un $29 \%$ siente que falta el apoyo del jefe, mientras que el $9 \%$ de las ocupaciones elementales siente que falta el apoyo de compañeros de trabajo. A continuación, las estadísticas completas (figura 4). Del total de la muestra evaluada, el 5\% percibe la falta de apoyo de los compañeros de trabajo y el $13 \%$ señala la ausencia de apoyo del jefe. 


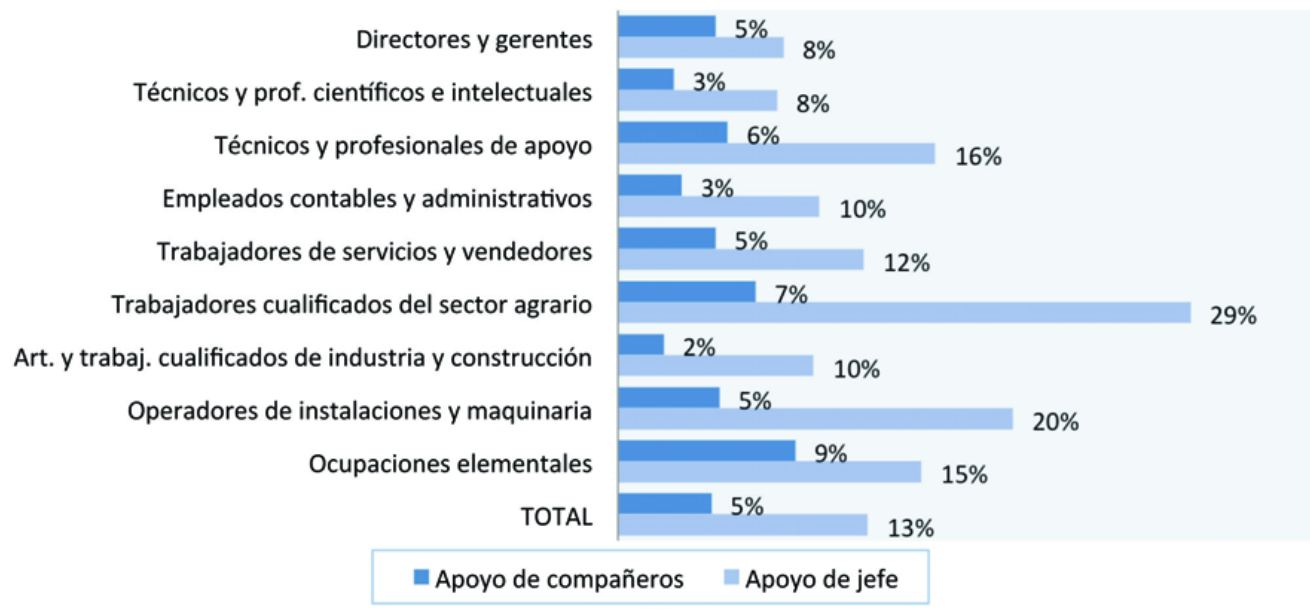

Figura 4. Falta de apoyo social en el trabajo (INSHT, 2017, p. 53)

\subsection{Percepción de la Inseguridad en el empleo}

Se mide respecto a la posibilidad que siente el trabajador de perder su empleo. Cabe mencionar que las estadísticas basadas en una muestra de 3.364 trabajadores, indican que el $30 \%$ de los trabajadores de hasta 34 años, perciben que tienen una alta probabilidad de perder su empleo en los próximos 6 meses, mientras que el $52 \%$ de los trabajadores de 50 años y más, manifiestan que no les sería fácil encontrar otro trabajo con un salario similar.

La figura 5 muestra las estadísticas completas, resaltando que la posibilidad de perder el empleo en los próximos 6 meses y la posibilidad no encontrar otro trabajo con salario similar, guardan una relación indirecta y directamente proporcional, respectivamente, respecto a la edad.

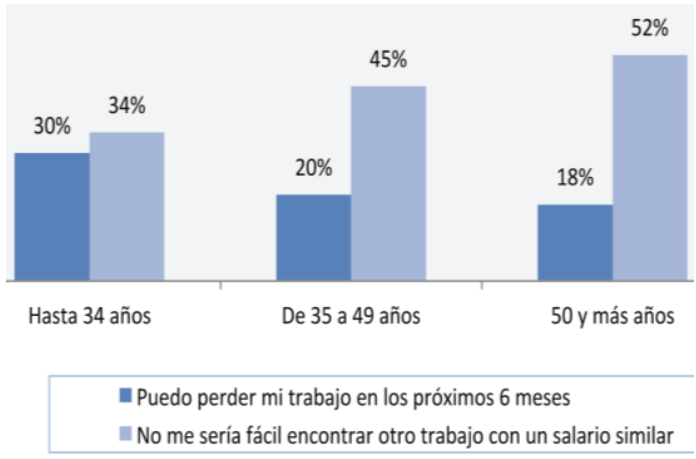

Figura 5. Parámetros de la percepción de la seguridad del trabajo (INSHT, 2017, p. 54)

\subsection{Situaciones de violencia, acoso o discriminación}

En lo que respecta a las situaciones de violencia, acoso o discriminación, las estadísticas expresan que "en 2015 al menos un 5\% de los 
trabajadores en España han sufrido algún tipo de discriminación" (INSHT, 2017, p. 53). No obstante, se expone una tabla con el porcentaje de violencia, acoso o discriminación que han sufrido los trabajadores, sobre una base de 3.364 trabajadores.

Tabla 1.

Colectivos expuestos a violencia, acoso o discriminación

\begin{tabular}{|c|c|c|c|}
\hline & Actividad económica & Ocupación & Total \\
\hline Discriminación por edad ${ }^{(1)}$ & $\begin{array}{c}\text { Industria (4\%) } \\
\text { Actividades administrativas, } \\
\text { auxiliares y financieras (4\%) }\end{array}$ & - & $2 \%$ \\
\hline Violencia física $^{(1)}$ & $\begin{array}{c}\text { Salud (5\%) } \\
\text { Administración pública y defensa (4\%) }\end{array}$ & $\begin{array}{c}\text { Trabajadores de servicios y } \\
\text { vendedores }(3 \%)\end{array}$ & $1 \%$ \\
\hline Acoso/intimidación ${ }^{(1)}$ & Salud $(7 \%)$ & $\begin{array}{c}\text { Trabajadores de servicios y } \\
\text { vendedores }(6 \%)\end{array}$ & $3 \%$ \\
\hline Ofensas verbales ${ }^{(2)}$ & Salud (15\%) & $\begin{array}{c}\text { Trabajadores de servicios y } \\
\text { vendedores (11\%) }\end{array}$ & $8 \%$ \\
\hline $\begin{array}{l}\text { Atención sexual no } \\
\text { deseada }^{(2)}\end{array}$ & Comercio y hostelería (3\%) & $\begin{array}{c}\text { Trabajadores de servicios y } \\
\text { vendedores }(3 \%)\end{array}$ & $1 \%$ \\
\hline Amenazas ${ }^{(2)}$ & $\begin{array}{c}\text { Administración pública y defensa (9\%) } \\
\text { Transporte }(7 \%) \\
\text { Actividades administrativas, auxiliares } \\
\text { y financieras }(6 \%)\end{array}$ & $\begin{array}{c}\text { Trabajadores de servicios y } \\
\text { vendedores }(7 \%)\end{array}$ & $4 \%$ \\
\hline
\end{tabular}

Fuente: Tomado de INSHT (2017, p. 55)

Una vez revisadas las estadísticas de los factores de riesgo psicosocial, podemos notar que los mismos, afectan a un porcentaje significativo de la población trabajadora que fue muestreada, por lo cual, es necesario evaluar en cada centro de trabajo si estos factores están presentes en la organización, para de esta forma, garantizar al trabajador un adecuado bienestar en el trabajo, derecho adquirido según normativas legales tanto nacionales, como internacionales.
Es por esto, que el desequilibro entre estos tres factores, da como resultado efectos perjudiciales para la salud de los trabajadores y el logro de los objetivos de la organización (Ceballos-Vásquez et al., 2015), por lo cual se vuelve imperiosa la necesidad de establecer en cada organización, una adecuada evaluación de los riesgos psicosociales que pueden estar afectando negativamente a los trabajadores y a la organización. 


\section{Conclusiones}

Se debe considerar que no todos los factores de riesgo psicosocial, están presentes en todos los puestos de trabajo, sino que existen puestos de trabajo en donde un factor puede estar más arraigado que otro; por ejemplo, el apoyo social de superiores, puede que no esté presente en trabajadores autónomos por la misma naturaleza del trabajo. Además, hay ciertos puestos de trabajo dónde un factor de riesgo psicosocial no se convierte en un aspecto negativo del puesto de trabajo, sino que puede ser un aspecto positivo del mismo (Ceballos-Vásquez et al., 2015).

\section{Bibliografía}

Bernal, D., Campos-Serna, J., Tobias, A., Vargas-Prada, S., Benavides, F. G., \& Serra, C. (2015). Work-related psychosocial risk factors and musculoskeletal disorders in hospital nurses and nursing aides: A systematic review and meta-analysis. International Journal of Nursing Studies, 52(2), 635648.

https://doi.org/10.1016/j.jjnurs tu.2014.11.003

Ceballos-Vásquez, P., RoloGonzález, G., HérnandezFernaud, E., Díaz-Cabrera,
D., Paravic-Klijn, T., \& BurgosMoreno, M. (2015). Psychosocial factors and mental work load: a reality perceived by nurses in intensive care units. Revista Latino-Americana de Enfermagem, 23(2), 315-322. https://doi.org/10.1590/01041169.0044 .2557

García, M. M., Iglesias, S., Saleta, M., \& Romay, J. (2016). Riesgos psicosociales en el profesorado de enseñanza universitaria: diagnóstico y prevención. Revista de Psicología del Trabajo y de las Organizaciones, 32(3), 173182.

https://doi.org/10.1016/j.rpto.2 016.07.001

Gil-Monte, P. R. (2009). Algunas razones para considerar los riesgos psicosociales en el trabajo y sus consecuencias en la salud pública. Rev. Esp Salud Pública, 2(83), 169173.

Guilguiruca, M., Meza, K., Góngora, R., \& Moya, C. (2015). Factores de riesgo psicosocial y estrés percibido en trabajadores de una empresa eléctrica en Chile. Medicina y seguridad del trabajo (Internet), 61(238), 57-67. https://doi.org/10.4321/S0465 -546 X2015000100006

INSHT. (2017). Encuesta Nacional de Condiciones de Trabajo. $20156^{\circ}$ EWCS - España. (J. Pinilla García, A. Almodóvar 
Molina, M. L. Galiana Blanco, \& M. Zimmermann Verdejo, Eds.). Madrid: Autor.

Martín-García, J., Luceño-Moreno, L., Talavera-Velasco, B., \& Jaén-Díaz, M. (2015). Factores de riesgo psicosocial en atención primaria: un estudio comparativo entre puestos. Atención Primaria, 47(9), 608-609. https://doi.org/10.1016/j.aprim .2015.01.004
Palomo-Vélez, G., Carrasco, J., Bastías, Á., Mendez, M. D., \& Jiménez, A. (2015). Factores de riesgo psicosocial y satisfacción laboral en trabajadoras estacionales de Chile. Rev Panam Salud Publica, 37(4/5), 301-307.

Real Decreto Ley 31/1995 de 8 de noviembre, de Prevención de Riesgos Laborales. Boletín Oficial del Estado, 209, de 10 de noviembre de 1995 